\title{
Realization of an Optical Indoor Positioning System Based on TFT Technology
}

\author{
István Gőzse, Alexandros Soumelidis, Bálint Vanek
}

\begin{abstract}
The aim of this paper is to present a novel indoor positioning system which is based on a unique idea. The proposed positioning system provides an alternative to currently existing positioning system. A new idea of optical sensing has been applied that in combination with specific analogue preprocessing and digital signal processing techniques is capable to determine the position and the orientation of objects equipped with special markers. The method is based on moving a pinhole between a marker and the photodiode on the detector. A prototype realization is also outlined, and experimental results are presented to confirm the applicability of the method.
\end{abstract}

\section{INTRODUCTION}

Single and cooperative control of unmanned vehicles is an intensive research field. Experimental validation of the control methods and algorithms that are developed gain crucial significance. For this purpose model vehicles are commonly used. A significant part of a model platform is some kind of positioning system that is capable of determining the position and orientation of the vehicles.

In the field of positioning several principles are used with different purpose. Indoor positioning systems can be categorized into two distinct groups. The first group tries to roughly localize the tracked object with the simplest method, so the basic aim is the location awareness. Good example on this is the Bluetooth based indoor positioning [9]. System like this can be used to track customers (who carry their mobile phone with Bluetooth) in a supermarket. The acquired information can be useful to evaluate the customer behaviour. Many other indoor positioning system address this problem [1] [2] [3]. This paper discusses a positioning system from the other main group of positioning, which focuses on the accurate spatial location and orientation determination, from a point of view of unmanned indoor vehicles like mobile robots or indoor quadrotors. In this field several different principles are used [4] [6] but the only widely used solution is camera based systems [10] The most advaced camera based systems are the so called motion capture (MOCAP) system, which was originally used in biomechanics and film industry. Nowadays MOCAP systems are also used in research of autonomous vehicles [5]. These systems - besides their advantages - suffer from several drawbacks, e.g. they usually require specifically designed laboratory with controlled ambient light. Furthermore a large amount of computational power is required to process the pictures provided by the cameras, as well as special optics are being used to correct imaging - these all make the camera based MOCAP system to be rather expensive, and inconvenient both in use and in development. A solution that possesses all the advantages of an optical MOCAP system, while being flexible enough to be realized at low cost, would be advantageous in the field of research of unmanned vehicles. The approach proposed in this paper tries to fulfill the above requirements. The main idea: a transparent graphic display realizing a variable pinhole camera is used as motion capture sensor to detect the position of one or more lighting markers.

\section{OPERATING PRINCIPLE}

The positioning system presented here uses active markers to mark the point what is under measurement. The marker is placed on the model vehicle which is traced by the system. LEDs are used as active markers. Their small size and low weight makes them ideal to be used on small sized model vehicles. In most of the cases the sensing element of an active marker is a camera but the recognition of the marker involves a very complicated image processing that is one of the greatest issue in camera based system; to avoid this problem the discussed system uses photodiode based optical sensing. By this means a rather reliable link can be established between the LED marker and the sensing system using adequate pulse modulation. Reliability can be achieved by using proper preamplification and signal processing discussed later in this paper. Using a photodiode as a detector - with proper signal processing -the disturbing ambient light can be eliminated during marker recognition. One photodiode alone is not sufficient in solving the problem as it detects only the existence of the light, deriving the arrival angle needs further elements. (Figure 2)

Considering a pinhole camera and a point light source the angle of arrival of the light source can be determined from the position of the image of the light source in the camera screen and the distance from hole to camera screen. (Figure 1. ) 


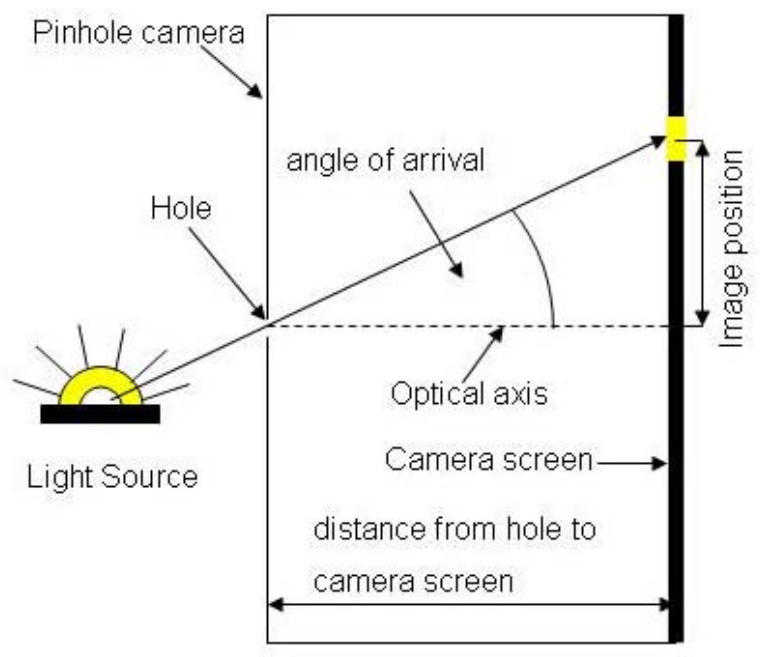

Figure 1: Angle of arrival determination with simple pinhole

camera.

To achieve a practical system one can reverse the angle of arrival determination process from a point of view of image and hole arrangement, in other words the image of the point light source on the screen stays still and the hole is moving. Considering a special pinhole camera which has a movable hole the spatial direction of the point light source can be determined if the image is staying still on the optical axis of the camera, and the hole moves according to the light source position (Figure 2. )

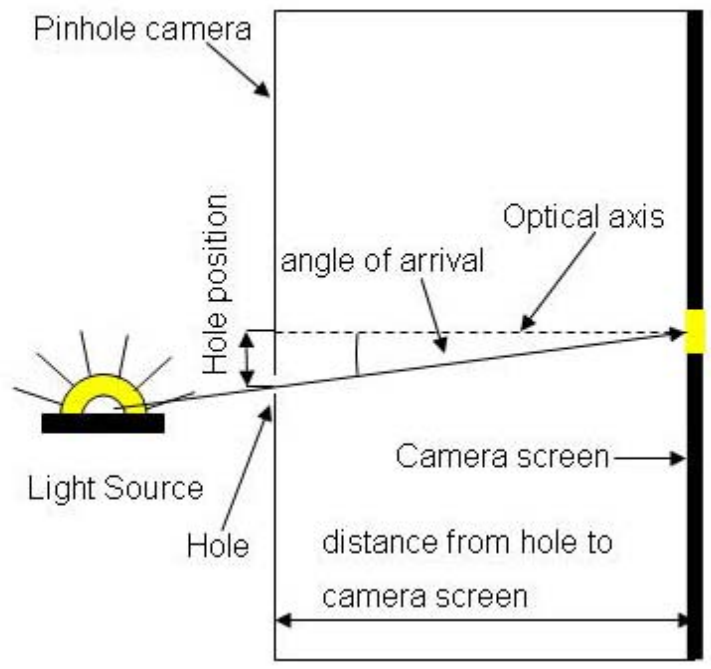

Figure 2: Angle of arrival determination with modified movable hole pinhole camera.

In this case the meaning of and the value of the angel of arrival has changed from the original concept, but the line of sight vector can be used to determine the location of the marker.
The key idea is that a pinhole camera equipped with movable hole can be realized with simple elements. It can be constructed by a TFT (thin-film-transistor) unit which makes the position determination possible by the displaying a specific picture in the image plane. The image is a completely black picture, with only one (or more) pixel nit illuminated. A regular TFT display can be disassembled to a tft unit and a backlight panel.(Figure 3. )

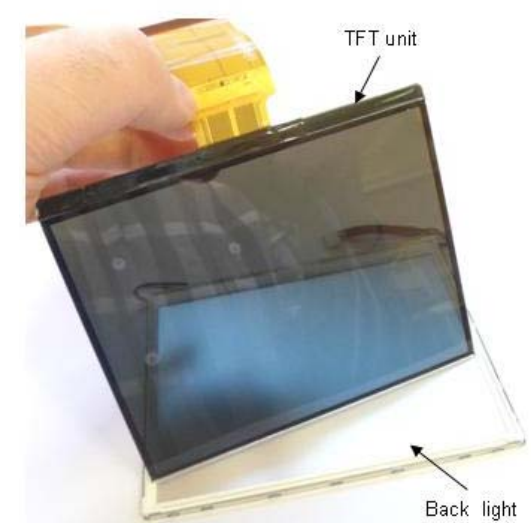

Figure 3: Disassambled TFT module.

If one uses only black and white colours in the image plane of the TFT display, the TFT unit can be interpreted as a special window that can be transparent or opaque according to the image displayed in it. This special window can be mounted in a distance in front of the photodiode which is placed in the middle of the camera screen. In this case driving every pixel of the TFT unit into the opaque state except leaving a small transparent patch (hole of the pinhole camera) on the line connecting the LED and the photodiode the coordinates of the patch indicate the direction of the marker relative to the photodiode. If the patch tracks the LED (marker), the instrument can maintain continuous direction measurements frame by frame that are displayed on TFT panel. The spatial position of a marker can be determined using geometrical methods by fitting two sensors, which are positioned far enough from each other (the principle of stereo vision is realized). Figure 4. shows the theoretical scheme of the positioning system.

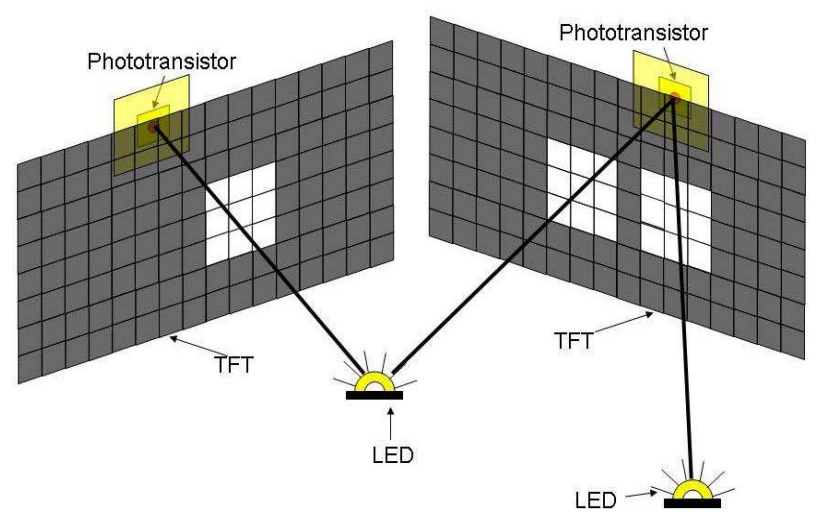

Figure 4: Theoretical scheme of the positioning system. 
To simplify the marker tracking task four independent photodiodes are used according to Figure 5. The photodiodes form a square shaped array where is a photodiode on each corner of the square. This sensor array is rotated 45 degree relative to the TFT pixel grid. Arrangement like this allows determining the heading of the marker under measurement.

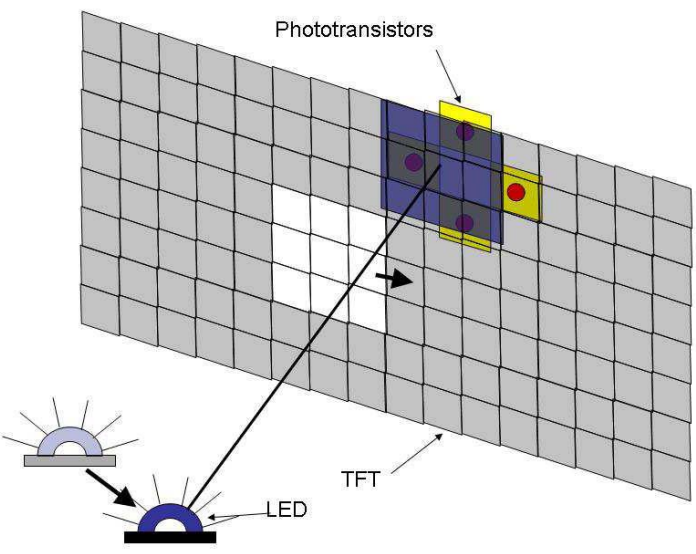

Figure 5 The LED tracking.

\section{INTRODUCTION OF THE PROTOTYPE}

To demonstrate the whole concept a prototype was built as it has been presented in Figure 6. The prototype consists three main parts, the TFT and its driving circuits, the light sensor module which contains the phototransistors, and the power supply module.

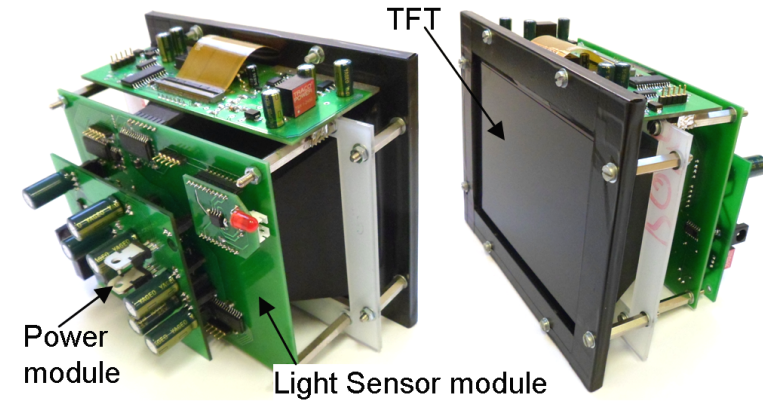

Figure 6: The prototype of the indoor positioning sensor.

The explode view (Figure 7.) of the positioning sensor reveals the realized construction of the above mentioned theoretical scheme. The phototransistor array is placed on the optical axis of the instrument; it can be seen in the middle of the light sensor module surrounded by the analogue amplifier circuits.

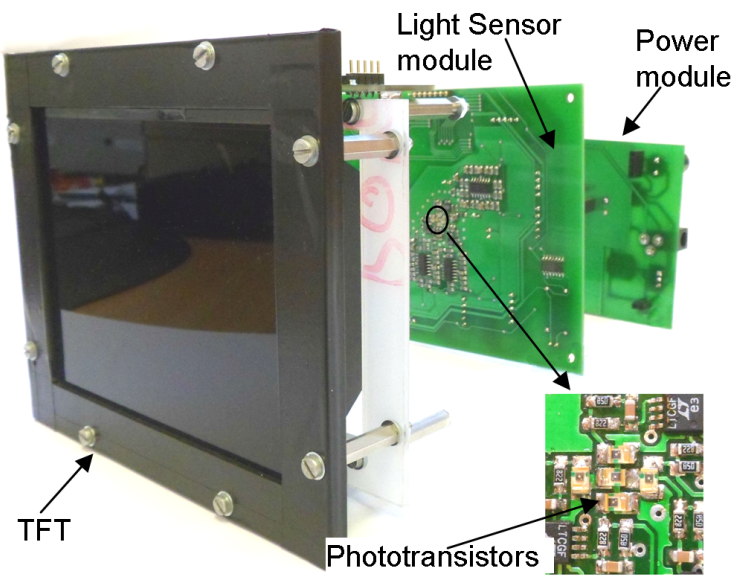

Figure 7: Explode view of the prototype

\section{A. Optical receiver}

The optical receiver is one of the most important parts of the positioning system.

The optical receiver of the indoor positioning system has to fulfill the following requirements:

1. Eliminate the effect of the ambient light caused by sunlight or lighting.

2. Measure the markers from a suitable distance.

3. Recognize the independent markers.

4. Measure a distant marker while there is an adjacent marker. (signal of adjacent marker can fade signal of distant marker)

The requirements of the optical sensor can be interpreted from a different point of view depending on whether the LED, the hole, and the phototransistor is on the line or not. This basically means luminance measurement of the LED and only the luminance of that particular LED should be measured. So the above mentioned requirements can be fulfilled with a customized photometer.

The receiver should discriminate multiple LEDs. A method that can be used to realize this is blinking of LEDs in different frequencies. This characterization was done by blinking. Each LED has its own unique blinking frequency what makes them different from other source of lights.

\section{B. Analogue circuits}

According to the above mentioned strategy the realized prototype uses analogue techniques to satisfy the requirement No 1. and No 2. The effect of the ambient light can be eliminated by using a high-pass preamplifier because in most cases the main frequency component of the ambient light is its DC component. The preamplifier is a crucial point of the analogue circuits because it basically influences the quality of the measured signal. The applied preamplifier [8] can be seen in the Figure 8.

The preamplifier has to be a high-pass filter because the disturbing ambient light can generate much greater input signal than the marker so without a high-pass preamplifier sufficient amplification can not be reached because the output of the preamplifier would saturate. 
Using a high-pass preamplifier with high pass second stage gives acceptable gain and filtering of the signal of the phototransistor. (block diagram in Figure 8. ) At the next step the signal is sampled by an A/D converter and the signal is processed with digital signal controller.

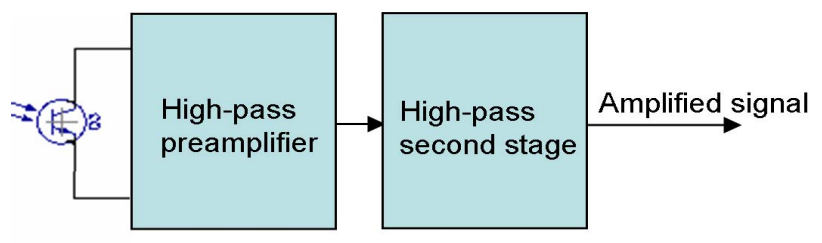

Figure 8: Analogue circuit semantic

\section{Digital processing of the amplified signal}

Requirements No 3 and No 4 can be fulfilled by using digital signal processing. Exploiting that each LED blinks in a given frequency one can take the discrete Fourier transformation of the signal. The frequency that belongs to the particular LED appears as a spike in the result of the transformation and the absolute value of that point is related to the luminance of the perceptible portion of the LED. So the luminance of a LED which blinks at a predefined frequency can be estimated with the following basic formula[7]:

$$
I_{L E D 1}=\left|\sum_{n=0}^{N-1} x_{n} \cdot e^{-j 2 \pi \frac{k}{N} n}\right|
$$

where $\mathrm{xn}$ is the measured signal ILED1 is the estimated luminance $\mathrm{N}$ is the number of sample in a window and " $\mathrm{k}$ " is an integer that corresponds to the frequency of the LED at the given sampling frequency. The above mentioned luminance value can be calculated with Fast Fourier transformation as well, but FFT calculates the whole transformation that can take more computation resources than calculating only those transformation points where the LED frequencies are presented. The number of the markers and the used number of the samples determine whether the FFT or row-by-row transformation takes less computation resources. The realized system uses the above mentioned formula. The measurement is special in a sense that the blinking frequency the time window and the sampling frequency can be determined during the design phase. This specialty allows choosing the measurement parameters in an effective way. It is an obvious choice that the time window is a multiple of the sample period and at the same time the time window is also a multiple of the blinking period. At these circumstances theoretically there is no spectral leakage between the frequencies of the markers. The prototype uses $1 \mathrm{msec}$ time window, $400 \mathrm{Ksamples} / \mathrm{sec}$ sampling rate, and the blinking frequencies of the markers is $10 \mathrm{kHz}, 12 \mathrm{kHz}, 14 \mathrm{kHz}$ etc. One can take advantage of this measurement setup in a different way. During each time window a sequence of samples is acquired, and since the time window is multiple of any blinking frequency one can apply a filter on the series of the samples with the same index number in consecutive time windows. This filter can significantly improve the quality of the luminance estimation. This also means if the time window is $1 \mathrm{msec}$ and the sampling rate is 400 Ksamples/sec four hundred filters have to be implemented, which would take a lot of computation resources. By choosing a moving average filter the computing needs can be reduced dramatically according to the following equations. At the i-th step the estimated luminance with $\mathrm{m}$ order moving average filter can be expressed as,

$$
I_{L E D 1}=\left|\sum_{n=0}^{N-1} e^{-j 2 \pi \frac{k}{N} n} \cdot\left(\frac{1}{m} \cdot \sum_{l=i}^{i-m} x_{n l}\right)\right| i \succ m
$$

where $\mathrm{xnl}$ is the $\mathrm{n}$-th element of the 1-th acquired sample sequence, ILED1 is the estimated luminance, $\mathrm{N}$ is the number of sample in a window " $\mathrm{k}$ " is an integer that corresponds to the frequency of the LED at the given sampling frequency. Obviously the above mentioned equation can be expressed as,

$$
I_{L E D 1}=\mid \frac{1}{m} \sum_{l=i}^{i-m}\left(\sum_{n=0}^{N-1} e^{-j 2 \pi \frac{k}{N} n} \cdot x_{n l}\right) \quad i \succ m
$$

The last equation means that it is not necessary to implement as many filters as many sample are in a time window only two filters have to be implemented; one for the real part of the result of the inner sum and one for the imaginary part. This solution saves a lot of computation resources and increases the quality of the luminance estimation. In real prototype the value of $m$ is sixteen, what gives a good tradeoff between noise filtering and bandwidth.

\section{EXPERIMENTAL RESULTS}

The performance of the prototype is demonstrated in two test scene. At the first test a fixed randomly placed LED is measured by the instrument. The series of the measurement can be seen in Figure 9. According to the diagram the measured deflection is one pixel most of the time, what means about 0.3-0.4 degree error..

Figure 9: Fixed marker measurement

The marker tracking capability is tested with the second test setup. The marker moves on a known route as the Figure
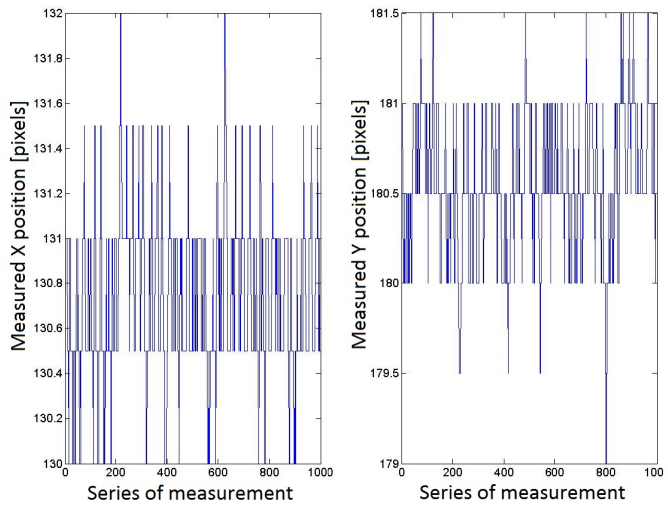

10. shows. 


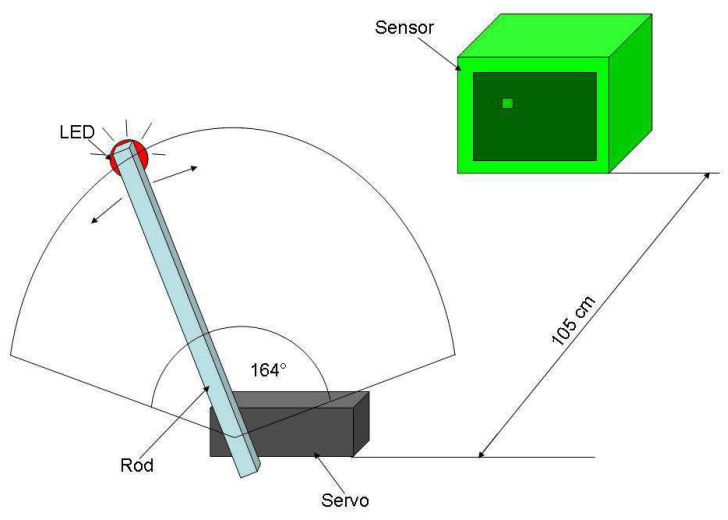

Figure 10 Test setup

The marker moves on a stick, which is swung by a servo motor, this means the marker stays on a fixed route while moving. The servo moves 164 degrees left-right with a 15.2 sec period. The stick's length is $27,5 \mathrm{~cm}$. The sensor is in distance $105 \mathrm{~cm}$ from the LED. The measured data and coordinates are compared with the previously calculated ones. The measured data and the calculated trajectory are shown in Figure 11.

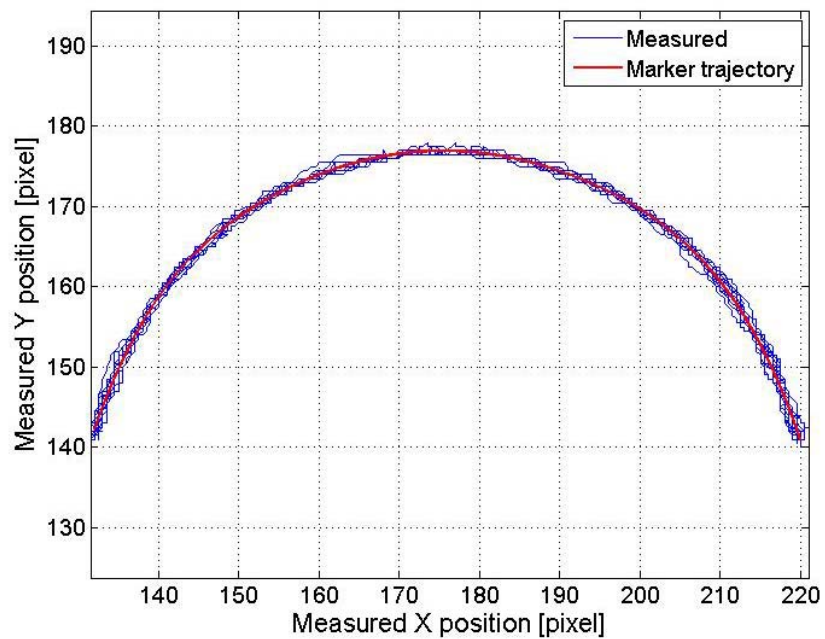

Figure 11: Measured moving marker track

The distance between the points and the theoretic curve is nearly one pixel most of the times.

To experiment the capabilities of the positioning system an autonomous indoor coaxial helicopter platform is realised equipped with the presented positioning system.

The overall system contains four sensors placed each corner of a four meter by four meter working area, and a modified RC model coaxial helicopter (Figure 12) which is controller by a computer.

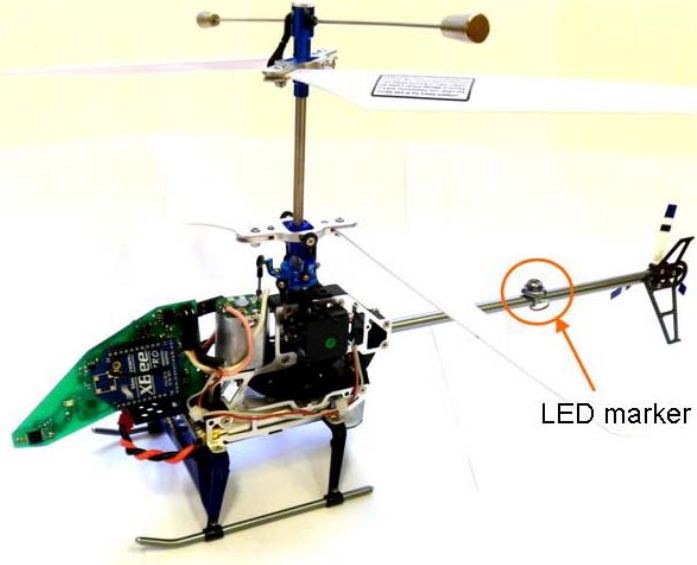

Figure 12: Autonomous coaxial helicopter

For test purpose the helicopter is controlled along an oval track. The following figures show one whole lap of the helicopter flight.

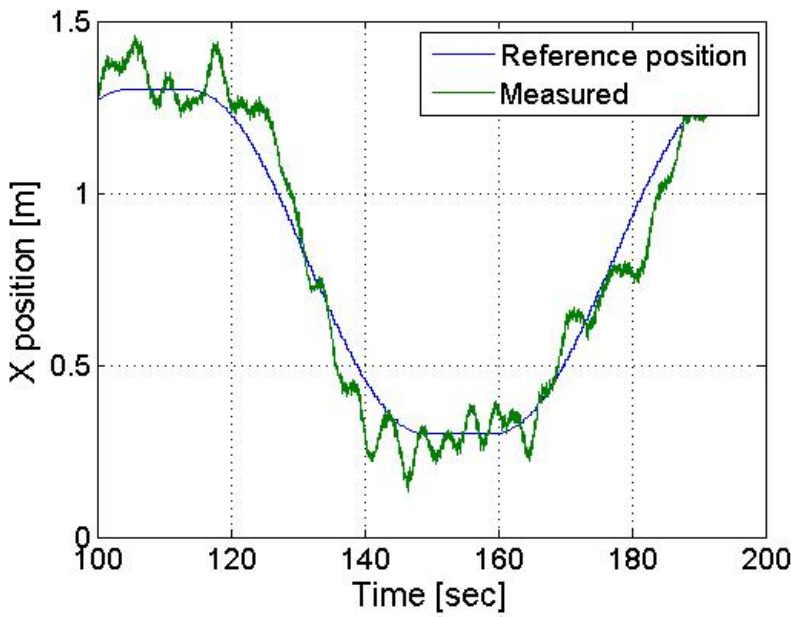

Figure 13: Measured X position

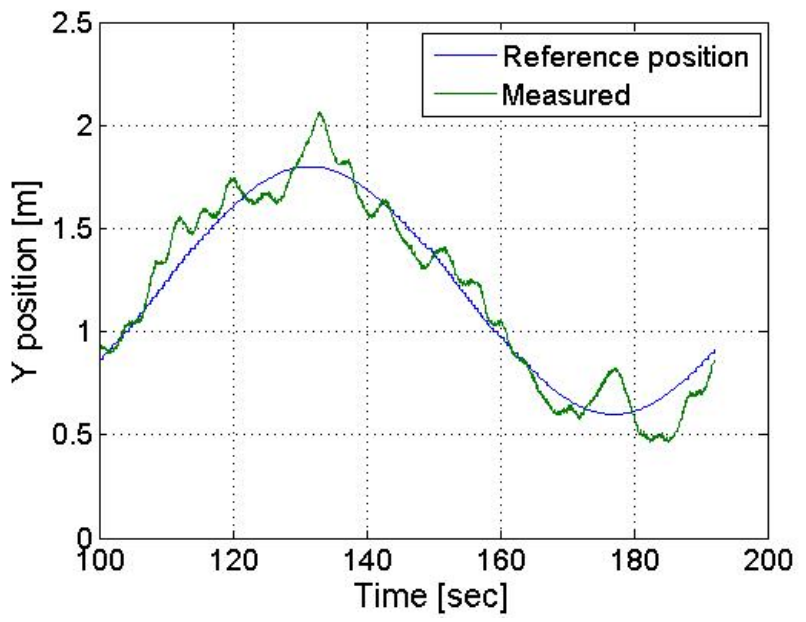

Figure 13: Measured Y position 


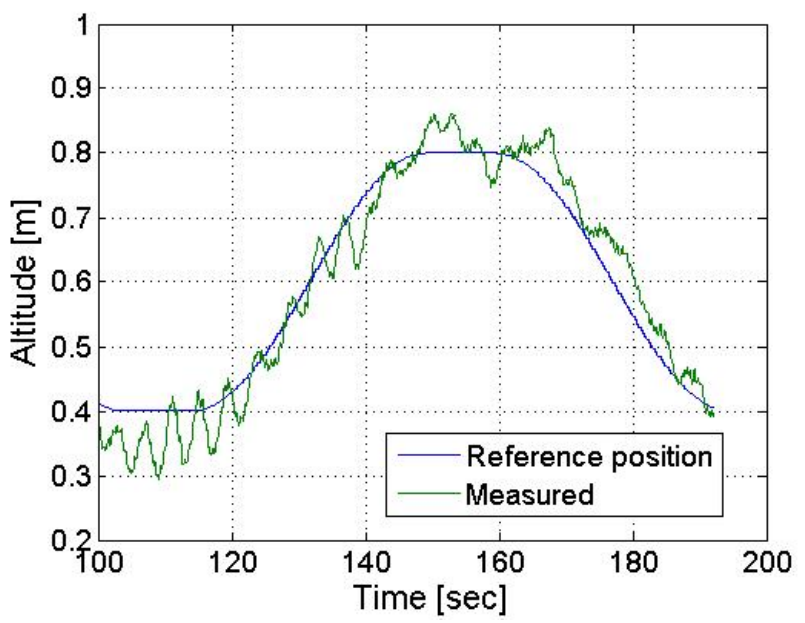

Figure 13: Measured altitude

Since one marker is mounted on the helicopter the orientation is controlled by on-board magnetic sensor.

\section{CONCLUSION}

The first part of the paper presents an alternative solution about an indoor positioning system which uses a transparent patch on a TFT unit to decode the relative direction of an LED. The greatest advantage of this solution is that the marker recognition is solved in a very robust and reliable way. This capability of recognition makes the system very simple and inexpensive solution compared to systems which have the same ability. As it was shown the system can track a marker what is moving on a predefined trajectory.

Important result is that the positioning system can support a complicated control platform like indoor autonomous helicopter test equipment.

\section{REFERENCES}

[1] Adrian David Cheok and Li Yue, A Novel Light-Sensor-Based Information Transmission System for Indoor Positioning and Navigation IEEE TRANSACTIONS ON INSTRUMENTATION AND MEASUREMENT, VOL. 60, 2011, pp. 290-299

[2] Ali Asghar Nazari Shirehjini, Abdulsalam Yassine, Shervin Shirmohammadi, An RFID-Based Position and Orientation Measurement System for Mobile Objects in Intelligent Environments, IEEE TRANS. ON INSTRUM. MEAS. VOL. 61, NO. 1, 2011, pp. 1664-1675

[3] Ali Asghar Nazari Shirehjini and Shervin Shirmohammadi , A High Precision Sensor System for Indoor Object Positioning and Monitoring, Distributed and Collaborative Virtual Environments Research Laboratory (Discover Lab) School of Information Technology and Engineering, University of Ottawa, Ottawa, Canada, 2009

[4] Ernesto Martín-Gorostiza, Fco Javier Meca, José Luis Lázaro Galilea, Eduardo Martos-Naya, Fernando B. Naranjo, and Óscar Esteban, Coverage-Mapping Method Based on a Hardware Model for MobileRobot Positioning in Intelligent Spaces, IEEE TRANSACTIONS ON INSTRUMENTATION AND MEASUREMENT, VOL. 59, 2010, pp. 266-282
[5] Guillaume Ducard and Raffaello D' Andrea, Autonomous Quadrotor Flight Using a Vision System And Accommodating Frames Misalignment, Industrial Embedded Systems, 2009, SIES

[6] Immanuel A. R. Ashokaraj, Peter M. G. Silson, Antonios Tsourdos, Member, IEEE, and Brian A. White, Robust Sensor-Based Navigation for Mobile Robots IEEE TRANSACTIONS ON INSTRUMENTATION AND MEASUREMENT, VOL. 58, 2009, pp. 551-556

[7] James V.Caddy, "Signal Processing”, McGraw-Hill, 1988

[8] John P Dakin, "Handbook of Optoelectronics" University of Southhampton, UK, 2006

[9] Josef Hallberg, Marcus Nilsson, Kare Synnes, Positioning with Bluetooth, Proceedings of the 10th International Conference on Telecommunications, 2003

[10] Rainer Mautz and Sebastian Tilch, Survey of Optical Indoor Positioning Systems, International Conference on Indoor Positioning and Indoor Navigation, 2011 